\title{
INTRODUCTION TO A QUASI-LINEAR POTENTIAL THEORY
}

\author{
ILPO LAINE
}

\section{Introduction}

The axiomatic theory of harmonic spaces is an important area of the linear potential theory investigated actively during the last decades. We may refer to Brelot [6], Bauer [1] and Constantinescu and Cornea [9] as standard references. Recently, considerable interest has been attracted by some non-linear questions reflecting ideas from the linear potential theory. We recall here Bertin [3]-[5], Bedford and Taylor [2] and the two articles of Constantinescu [7], [8] devoted to the nonlinear Dirichlet problem.

Granlund, Lindqvist and Martio recently investigated ([10], [11]) quite a general class of variational integrals in $\boldsymbol{R}^{n}$ from a potential theoretic point of view. Remarkably, their presentation is, in some sense, quite close to the usual linear theory. Lehtola [12] also developed a related axiomatic system, essentially corresponding to the linear theory of Brelot spaces.

This article was inspired by the work of Granlund, Lindqvist and Martio. Our aim has been to develop non-linear axioms describing the behavior of variational super-F-extremals, but in the spirit of Constantinescu and Cornea [9]. In Section 2, we give the axioms and state basic topological properties. Some examples will be also included in Section 2. In Section 3, we develop the elementary quasi-linear theory. Perhaps we should point out here the essential problem remaining open in this article, namely the counterpart to the Poisson modification. This carries through in the regular case, but remains open in general. Our final Section 4 is devoted to the basic theory of quasi-linear superharmonic functions and potentials. Evidently, the quasilinear theory developed in this article may be pursued further. Specially, the open problem about the Poisson modification should be resolved. This plan of research is under preparation and a more complete presentation of the quasi-linear theory will appear elsewhere.

Finally, I wish to express my gratitude to Olli Martio for many valuable discussions directed to non-linear phenomena in potential theory.

This research has been done in the frame of a research project supported in part by the Academy of Finland. 


\section{Quasi-linear axioms and basic examples}

Let $X$ be a locally compact topological space and let a sheaf $\mathscr{U}$ on $X$ of lower semicontinuous, hence lower finite numerical functions called a hyperharmonic sheaf be given. If $u \in \mathscr{U}(U)$, it is called hyperharmonic on $U$. The map $U \mapsto \mathscr{U}(U) \cap(-\mathscr{U}(U))$ also defines a sheaf of functions on $X$ called the harmonic sheaf $\mathscr{H}_{\mathscr{U}}$ defined by the hyperharmonic sheaf $\mathscr{U}$. We say that $u \in-\mathscr{U}(U)$ is hypoharmonic on $U$ and $h \in \mathscr{H}_{\mathscr{U}}(U)$ is harmonic on $U$. We assume the following

Axiom of quasi-linearity: The hyperharmonic sheaf $\mathscr{U}$ contains a non-empty subsheaf $\mathscr{V} \subset \mathscr{H}_{\mathscr{U}}$ such that for every open set $U \subset X$,

(1) $\alpha v \in \mathscr{V}(U)$ for every $v \in \mathscr{V}(U)$ and every $\alpha \in \boldsymbol{R}$,

(2) $u+v \in \mathscr{U}(U)$ for every $u \in \mathscr{U}(U)$ and every $v \in \mathscr{V}(U)$.

Before proceeding, we remark that the axiom of quasi-linearity trivially implies that the constant function 0 is harmonic on any open set $U \subset X$ and, in fact, $0 \mid U \in \mathscr{V}(U)$.

An open set $U \subset X$ is now called an MP-set (relative to $\mathscr{U}$ ), if $u \geqq v$ holds for any lower bounded hyperharmonic function $u \in \mathscr{U}(U)$ and any upper bounded hypoharmonic function $v \in-\mathscr{U}(U)$ as soon as

(1) $\lim \inf _{U \ni x \rightarrow y} u(x) \geqq \lim \sup _{U \ni x \rightarrow y} v(x)$ for every $y \in \partial U$ and

(2) there exists a compact set $K(u, v)$ in $X$ such that $u \geqq v$ holds on $U \backslash K(u, v)$.

Let now $U$ be an MP-set and let $f: \partial U \rightarrow \overline{\boldsymbol{R}}$ be a given boundary function. We consider the family $\overline{\mathscr{U}}_{f}(U) \subset \mathscr{U}(U)$ of functions defined by the following conditions:

(1) every function $u \in \overline{\mathscr{U}}_{f}(U)$ is lower bounded,

(2) for every function $u \in \bar{U}_{f}(U)$ there corresponds a compact set $K(u)$ in $X$ such that $u$ is positive in $U \backslash K(u)$,

(3) for every function $u \in \overline{\mathscr{U}}_{f}(U)$ and every point $y \in \partial U$ we have $\lim \inf _{U \ni x \rightarrow y} u(x) \geqq f(y)$.

We also consider the family $\underline{\mathscr{U}}_{f}(U)=-\overline{\mathscr{U}}_{-f}(U)$. Of course, $u \in \underline{\mathscr{U}}_{f}(U)$ if and only if $u \in-\mathscr{U}(U)$ and (1) $u$ is upper bounded, (2) there exists a compact set $K(u)$ in $X$ such that $u$ is negative in $U \backslash K(u)$ and (3) for every point $y \in \partial U$ we have $\lim \sup _{U \ni x \rightarrow y} u(x) \leqq f(y)$. Defining

$$
\bar{H}_{f}^{U}:=\inf \overline{\mathscr{U}}_{f}(U), \quad \underline{H}_{f}^{U}:=\sup \underline{\mathscr{U}}_{f}(U)
$$

we easily verify the following elementary

Lemma 2.1. Let $U$ be an MP-set and let $f: \partial U \rightarrow \overline{\boldsymbol{R}}, g: \partial U \rightarrow \overline{\boldsymbol{R}}$ be given boundary functions. Then

$$
\begin{gathered}
-\bar{H}_{f}^{U}=\underline{H}_{-f}^{U}, \\
\quad \underline{H}_{f}^{U} \leqq \bar{H}_{f}^{U}, \\
f \leqq g \quad \text { implies } \quad \bar{H}_{f}^{U} \leqq \bar{H}_{g}^{U} \text { and } \underline{H}_{f}^{U} \leqq \underline{H}_{g}^{U} .
\end{gathered}
$$


Moreover, if $U$ is relatively compact in $V \subset X$ and $v \in \mathscr{V}(V)$, then

$$
\bar{H}_{f+v}^{U}=\bar{H}_{f}^{U}+v, \quad \underline{H}_{f+v}^{U}=\underline{H}_{f}^{U}+v .
$$

Proof. The first three assertions are trivial. To prove the last one, take $u \in \overline{\boldsymbol{U}}_{f}(U)$. Then $u+(v \mid U)$ is lower bounded and

$$
\liminf _{U \ni x \rightarrow y}(u(x)+v(x))=\liminf _{U \ni x \rightarrow y} u(x)+v(y) \geqq f(y)+v(y) .
$$

Hence $u+v \in \overline{\mathscr{U}}_{f+v}(U)$ and therefore $\bar{H}_{f+v}^{U} \leqq \bar{H}_{f}^{U}+v$. Since $-v \in \mathscr{V}(V)$, we also get $\bar{H}_{f}^{U}=\bar{H}_{(f+v)-v}^{U} \leqq \bar{H}_{f+v}^{U}-v$. The last assertion now follows.

A numerical boundary function $f: \partial U \rightarrow \bar{R}$ is called resolutive (relative to $\mathscr{U}$ ), if $\bar{H}_{f}^{U} \in \mathscr{H}_{\mathscr{U}}(U), \underline{H}_{f}^{U} \in \mathscr{H}_{\mathscr{U}}(U)$ and $\bar{H}_{f}^{U}=\underline{H}_{f}^{U}\left(=: H_{f}^{U}\right)$. Determining $H_{f}^{U}$ for a given boundary function $f$ is called the quasi-linear Dirichlet problem (in the PerronWiener-Brelot sense). An MP-set $U \subset X$ is called resolutive (relative to $\mathscr{U}$ ), if every function $f \in \mathscr{K}(\partial U)$ is resolutive.

Remark. If $f: \partial U \rightarrow \overline{\boldsymbol{R}}$ is resolutive, $U$ is relatively compact in $V \subset X$ and $v \in \mathscr{V}(V)$, then clearly $f+v$ is resolutive. However, the functional $f \mapsto H_{f}^{U}(x)$ is not necessarily a linear functional. This implies that in the quasi-linear theory there does not exist a harmonic measure in the usual sense of the linear theory.

Lemma 2.2. Let $U$ be a relatively compact, resolutive set. If $h$ is harmonic on an open set $V$ containing cl $U$, then $\underline{H}_{h}^{U}=\bar{H}_{h}^{U}=h$ on $U$. Moreover, if $u$ is hyperharmonic on $V$, then $\underline{H}_{u}^{U} \leqq u$.

Proof. To prove the first assertion, observe that $h \mid U$ is bounded and that $\lim _{U \ni x \rightarrow y} h(x)=h(y)$ holds for every boundary point $y \in \partial U$. Hence $h \in \overline{\mathscr{U}}_{h}(U)$ and $h \in \underline{U}_{h}(U)$. Therefore $h \leqq \underline{H}_{h}^{U} \leqq \bar{H}_{h}^{U} \leqq h$.

If now $u$ is hyperharmonic on $V$, then $u \in \overline{\mathscr{U}}_{u}(U)$ and therefore $\underline{H}_{u}^{U} \leqq \bar{H}_{u}^{U} \leqq u$.

We now proceed to the remaining axioms:

Axiom of resolutivity: The open sets resolutive relative to the hyperharmonic sheaf $\mathscr{U}$ form a base for the topology of $X$.

We recall that a sheaf $\mathscr{F}$ is degenerated at $x$, if every function $\varphi \in \mathscr{F}(U), U$ open and contains $x$, satisfies $\varphi(x)=0$. Otherwise $\mathscr{F}$ is non-degenerated at $x$.

Axiom of quasi-linear positivity: The quasi-linear subsheaf $\mathscr{V}$ determined by the axiom of quasi-linearity is non-degenerated at every point $x \in X$.

Axiom of completeness: A lower semicontinuous function $u: U \rightarrow(-\infty,+\infty]$ on an open set $U$ is hyperharmonic on $U$, if for every relatively compact, resolutive set $V$ such that $\mathrm{cl} V \subset U$, the inequality $H_{u}^{V} \leqq u$ holds.

Clearly, the axiom of completeness contains an if and only if-condition. This follows immediately from Lemma 2.2 . 
Axiom of convergence: The harmonic sheaf $\mathscr{H}_{\mathfrak{U}}$ defined by the hyperharmonic sheaf U possesses the Bauer convergence property; see [9], p. 9.

Definition 2.3. A locally compact space $X$ together with a hyperharmonic sheaf $\mathscr{U}$ is called a quasi-linear harmonic space, if the axioms of quasi-linearity, quasi-linear positivity, convergence, resolutivity and completeness hold. Quasi-linear harmonic spaces induced into open sets $U$ of $X$ may be called quasi-linear harmonic subspaces.

Remark 2.4. Some basic topological properties of harmonic spaces will be derived without appealing to linearity. Hence we may apply the usual proofs given in the linear theory to see that a quasi-linear harmonic space $X$ is completely regular and locally connected. Also, all points of $X$ are non-isolated. Moreover, the following proposition follows by the same reasoning as in the siandard linear theory:

Proposition 2.5. Let $\mathscr{F}$ be an upper directed family of harmonic functions on an open set $U \subset X$. If sup $\mathscr{F}$ is locally bounded, it is harmonic on $U$. Similarly, if the Doob convergence property, see [9], p. 9, holds for $\mathscr{H}_{\mathscr{U}}$ and $\sup \mathscr{F}$ is finite on a dense subset of $U$, then $\sup \mathscr{F}$ is harmonic on $U$.

Example 2.6. This example deals with the variational integrals mentioned in the introduction. Consider the euclidean space $\boldsymbol{R}^{n}$ and the sheaf $\mathscr{U}$ on $\boldsymbol{R}^{n}$ defined by the super-F-extremals in the sense of [10]. The variational kernel $F: \boldsymbol{R}^{n} \times \boldsymbol{R}^{n} \rightarrow \boldsymbol{R}$ has to satisfy the conditions (a), ( $\left.\mathrm{b}^{\prime}\right),(\mathrm{c})$ and (d) in [10], p. 48. We also recall that $u$ is a super-F-extremal, i.e., $u \in \mathscr{U}(U)$, if $h \leqq u$ on $\partial D$ implies $h \leqq u$ on $D$ for every function $h \in \mathscr{H}_{\mathscr{U}}(U) \cap \mathscr{C}(\mathrm{cl} D)$ and every regular domain $D$ relatively compact in $U$, regular meaning here a bounded domain with no boundary components consisting of one point only. By [10], Lemma 5.23, $\mathscr{U}$ is, in fact, a sheaf. Taking now as $\mathscr{V}$ the subsheaf of constant functions, the axiom of quasi-linearity is satisfied by [10], Lemma 5.2. A regular domain is an MP-set by [11], Lemma 2.3, and resolutive (in fact, regular in the Dirichlet problem sense) by [13], Theorem 3.10. The axiom of quasi-linear positivity holds trivially and the axiom of Bauer convergence by [10], Theorem 4.22. To verify the axiom of completeness, let $u: U \rightarrow(-\infty,+\infty]$ satisfy $\underline{H}_{u}^{V} \leqq u$ for every regular set $D$ relatively compact in $U$, and let $h \in \mathscr{H}_{\mathscr{U}}(U) \cap \mathscr{C}(\mathrm{cl} D)$ satisfy $h \leqq u$ on $\partial D$. Then $h=H_{h}^{V}=\underline{H}_{h}^{V} \leqq \underline{H}_{u}^{V} \leqq u$ holds on $D$ and the axiom of completeness follows.

Example 2.7. This example is due to Martio [a private communication]. Consider $X=\left\{(x, y) \in \boldsymbol{R}^{2} \mid x y=0, y \geqq 0\right\}$. The open intervals $I=(a, b) \times\{0\}$ and $I=\{0\} \times$ $(a, b)$ for $a, b \in \boldsymbol{R}, a<b, 0 \notin(a, b)$, and the neighbourhoods $T=(-t, t) \times[0, t)$ of the origin for $0<t<+\infty$ together form a base for the topology of $X$. We now define $\mathscr{H}(U)$ for open sets $U \subset X$ as follows:

(i) $h$ is linear on $U$ wherever $U$ is of the form $I$; 
(ii) $h(0)=(\max h(\partial U)+\min h(\partial U)) / 2$ and $h$ is linear on the intervals from the origin to the boundary points of $U$ whenever $U$ is of the form $T$;

(iii) $h \mid T \in \mathscr{H}(T)$ for $T=(-t, t) \times[0, t), t=\min (-a, b, c, 1)$, and $h$ is linear on the intervals (respectively half-lines) from the origin to the boundary points (respectively natural ideal boundary points) of $U$ whenever $U=(a, b) \times[0, c)$ for $-\infty \leqq a<0<b \leqq+\infty$ and $0<c \leqq+\infty$;

(iv) $h \mid U_{\alpha} \in \mathscr{H}\left(U_{\alpha}\right)$ for every component $U_{\alpha}$ of $U$ whenever $U$ is non-connected.

Defining now $u \in \mathscr{U}(U)$ by the comparison principle given in Example 2.6, i.e., $h \leqq u$ on $\partial V$ implies $h \leqq u$ on $V$ for every domain $V$ relatively compact in $U$, we get a sheaf $\mathscr{U}$ on $X$. It is easily verified that $(X, \mathscr{U})$ is a quasi-linear harmonic space in the sense of Definition 2.3.

\section{Elementary properties of quasi-linear harmonic spaces}

Lemma 3.1. Let $V$ be an open set such that there exists a strictly positive function $v_{0} \in \mathscr{V}(V)$. If $U$ is an MP-set relatively compact in $V$ and $\left(f_{\alpha}\right)_{\alpha \in I}$ is an upper directed family of lower semicontinuous functions on $\partial U$, then

$$
\underline{H}_{\alpha \in I}^{U} \sup _{\alpha}=\sup _{\alpha \in I} \underline{H}_{f_{\alpha}}^{U} .
$$

Proof. It suffices to prove the inequality $\underline{H}_{\sup f_{\alpha}}^{U} \leqq \sup \underline{H}_{f_{\alpha}}^{U}$. Take an arbitrary $v \in \underline{\mathscr{U}}_{\sup f_{\alpha}}^{I I}$ and extend $v$ into $\mathrm{cl} U$ by the upper limit. The extended function will be also denoted by $v$. Consider first a point $y \in \partial U$ such that $\sup f_{\alpha}(y)<+\infty$. Then

$$
v(y)=\limsup _{U \ni x \rightarrow y} v(x) \leqq \sup f_{\alpha}(y)<\frac{1}{2} \varepsilon v_{0}(y)+\sup f_{\alpha}(y) .
$$

Choosing now $\alpha_{y} \in I$ such that $\sup f_{\alpha}(y)<f_{\alpha_{y}}(y)+\frac{1}{2} \varepsilon v_{0}(y)$ we get

$$
v(y)-f_{\alpha_{y}}(y)-\varepsilon v_{0}(y)<0 \text {. }
$$

If now $y \in \partial U$ satisfies $\sup f_{\alpha}(y)=+\infty$, we again find $\alpha_{y} \in I$ such that the inequality (3.1) holds, since $v$ is upper bounded. Since $v-f_{\alpha_{y}}-\varepsilon v_{0}$ is upper semicontinuous on $\partial U$, the set

$$
\left\{z \in \partial U \mid v(z)-f_{\alpha_{y}}(z)-\varepsilon v_{0}(z)<0\right\}
$$

is open. Since $\partial U$ is compact and $\left(f_{\alpha}\right)_{\alpha \in I}$ is upper directed, we may apply a standard compactness argument to find an $\alpha_{0} \in I$ such that $v<f_{\alpha_{0}}+\varepsilon v_{0}$ holds on the whole boundary $\partial U$. Therefore $v \in \underline{U}_{f_{\alpha_{0}}+\varepsilon v_{0}}^{U}$ and by Lemma 2.1(4) we obtain

Hence

$$
v \leqq \underline{H}_{f_{\alpha_{0}}}^{U}+\varepsilon v_{0}=\underline{H}_{f_{\alpha_{0}}}^{U}+\varepsilon v_{0} \leqq \sup \underline{H}_{f_{\alpha}}^{U}+\varepsilon v_{0} .
$$

$$
\underline{H}_{\sup f_{\alpha}}^{U} \leqq \sup \underline{H}_{f_{\alpha}}^{U}+\varepsilon v_{0},
$$

and the assertion follows by $\varepsilon \rightarrow 0$. 
Remark. The preceding lemma seems to be an important device to overcome some difficulties which come from the fact that the functional $f \mapsto H_{f}^{U}(x)$ is not linear in general. It is reasonable to shorten the notation by saying that an open set $U$ is sufficiently small, if $\mathrm{cl} U$ is contained in an open set $V$ such that there exists a strictly positive function $v \in \mathscr{V}(V)$, see also [8], p. 58.

Proposition 3.2. Let $u_{1}, u_{2}$ be hyperharmonic on an open set $U$ and let $\left(u_{\alpha}\right)_{\alpha \in I}$ be an upper directed family of hyperharmonic functions on $U$. Then $\inf \left(u_{1}, u_{2}\right)$ and $\sup u_{\alpha}$ are hyperharmonic on $U$.

Proof. The first assertion follows immediately from the axiom of completeness.

To prove the second assertion, let $V$ be a resolutive set sufficiently small and relatively compact in $U$. If $W$ is a resolutive set relatively compact in $V$, then

$$
\underline{H}_{\sup u_{\alpha}}^{W}=\sup \underline{H}_{u_{\alpha}}^{W} \leqq \sup u_{\alpha}
$$

by Lemma 3.1. Hence $\sup u_{\alpha}$ is hyperharmonic on $V$ by the axiom of completeness. By the sheaf property of hyperharmonic functions (and the axiom of quasi-linear positivity), $\sup u_{\alpha}$ is hyperharmonic on $U$.

Proposition 3.3. Let $V$ be a relatively compact, resolutive and sufficiently small set and let $f: \partial V \rightarrow \boldsymbol{R}$ be a bounded lower semicontinuous boundary function. Then $\underline{H}_{f}^{V}$ is harmonic on $V$. If $f: \partial V \rightarrow \overline{\boldsymbol{R}}$ is lower semicontinuous, then $\underline{H}_{f}^{V}$ is hyperharmonic on $V$. Finally, if $W$ is resolutive and relatively compact in $V$, then

$$
\underline{H}_{\underline{H}_{f}^{V}}^{W}=\left(\underline{H}_{f}^{V}\right) \mid W .
$$

Proof. (1) Let $f$ be a bounded lower semicontinuous boundary function and denote

$$
\mathscr{F}:=\left\{H_{g}^{V} \mid g \leqq f, \quad g \in \mathscr{C}(\partial V)\right\} .
$$

Clearly $\mathscr{F}$ is an upper directed family of harmonic functions on $V$ and

$$
\sup \mathscr{F}=\sup H_{g}^{V}=\underline{H}_{\sup g}^{V}=\underline{H}_{f}^{V}
$$

by Lemma 3.1. Since $f \leqq \alpha$ for some real number $\alpha, \underline{H}_{f}^{V} \leqq \underline{H}_{\alpha}^{V}$ is locally upper bounded, since $H_{\alpha}^{V}$ is a harmonic function on $V$ by the axiom of resolutivity. By Proposition $2.5, \underline{H}_{f}^{V}$ is harmonic on $V$.

(2) Let $f$ be a lower semicontinuous boundary function. Since $f$ is lower bounded, $\inf (f, n)$ is bounded and lower semicontinuous for every $n \in N$. Since $f=\sup _{n} \inf (f, n)$ and $(\inf (f, n))_{n \in N}$ is an increasing sequence, we may apply Lemma 3.1 to obtain

$$
\underline{H}_{f}^{V}=\sup _{n} \underline{H}_{\inf (f, n)}^{V} .
$$

Since $\left(\underline{H}_{\mathrm{inf}(f, n)}^{V}\right)_{n \in N}$ is an increasing sequence of harmonic functions on $V, \underline{H}_{f}^{V}$ is hyperharmonic by Proposition 3.2. 
(3) To prove the last assertion, we denote $f_{n}:=\inf (f, n)$ for $n \in N$. Then

$$
\underline{H}_{\underline{H}_{n}}^{W}=\underline{H}_{f_{n}}^{V}
$$

holds on $W$ by Lemma 2.2, since $\underline{H}_{f_{n}}^{V}$ is harmonic on $V$ by the first part of this proof. Applying Lemma 3.1 again, we obtain

$$
\underline{H}_{f}^{V}=\underline{H}_{\sup f_{n}}^{V}=\sup \underline{H}_{f_{n}}^{V}=\sup \underline{H}_{\underline{H}_{n}}^{W}=\underline{H}_{\sup \underline{\underline{f}}_{f_{n}}^{V}}^{W}=\underline{H}_{\underline{H}_{\text {sup } f_{n}}^{V}}^{W}=\underline{H}_{\underline{H}_{f}^{V}}^{W}
$$

on $W$.

Proposition 3.4. The components of a resolutive set are resolutive.

Proof. Let $U$ be a resolutive set and $V$ be a component of $U$. We have to prove first that $V$ is an MP-set. Let $u$ (respectively $v$ ) be a lower bounded hyperharmonic (respectively an upper bounded hypoharmonic) function on $V$ such that

$$
\liminf _{V \ni x \rightarrow y} u(x) \geqq \limsup _{V \ni x \rightarrow y} v(x)
$$

holds for every $y \in \partial V$ and that $u \geqq v$ on $V \backslash K(u, v)$ for some compact set $K(u, v)$ in $X$. We define $u^{\prime} \in \mathscr{U}(U)$, respectively $v^{\prime} \in-\mathscr{U}(U)$ by setting $u^{\prime} \mid V=u$, $u^{\prime} \mid(U \backslash V)=0$, respectively $v^{\prime}\left|V=v, v^{\prime}\right|(U \backslash V)=0$. Since $U$ is an MP-set, $u^{\prime} \geqq v^{\prime}$ on $U$, hence $u=u^{\prime} \geqq v^{\prime}=v$ holds on $V$.

Since $X$ is completely regular, see Remark 2.4 , the assertion now follows by the same reasoning one has to apply in the linear theory of harmonic spaces.

Corollary 3.5. The open connected sets resolutive relative to the hyperharmonic sheaf $\mathscr{U}$ form a base for the topology of $X$.

\section{Superharmonic functions and potentials}

Definition 4.1. A hyperharmonic function $u$ on an open set $U \subset X$ is called superharmonic on $U$, if for every resolutive set $V$ relatively compact in $U$ and suffciently small, the lower Dirichlet solution $\underline{H}_{u}^{V}$ is harmonic on $V$.

The family of superharmonic functions on $U$ will be denoted by $\mathscr{S}(U)$.

Lemma 4.2. A superharmonic function $u \in \mathscr{S}(U)$ is finite on a dense subset of $U$.

Proof. Suppose there exists an open set $V \subset U$ such that $u \mid \mathrm{cl} V=+\infty$. We may assume that $\mathrm{cl} V \subset U$ and that $V$ is resolutive and sufficiently small. Let $v_{0}$ be a strictly positive function in a neighbourhood $(\subset U)$ of $V$. Clearly, we have $\lambda v_{0} \in \underline{\mathscr{U}}_{u}(V)$ for all $\lambda>0$. Hence $\underline{H}_{u}^{V} \geqq \lambda v_{0}$ for all $\lambda>0$ and we get a contradiction $H_{u}^{V}=+\infty$. 
Theorem 4.3. A quasi-linear harmonic space admits the Doob convergence property if and only if every function hyperharmonic on an open set is superharmonic as soon as it is finite on a dense subset.

Proof. Let first the Doob convergence property hold on a quasi-linear harmonic space $X$, let $u \in \mathscr{U}(U)$ be finite on a dense set and let $V$ be resolutive, sufficiently small and relatively compact in $U$. Denoting

$$
\mathscr{F}:=\{f \in \mathscr{C}(\partial V) \mid f \leqq u \text { on } \partial V\}
$$

we have $u=\sup \mathscr{F}$ on $\partial V$. For every $f \in \mathscr{F}, \underline{H}_{f}^{V}$ is harmonic and, by Lemma 3.1,

$$
\sup \underline{H}_{f}^{V}=\underline{H}_{\text {sup } \mathscr{F}}^{V}=\underline{H}_{u}^{V} \leqq u
$$

on $V$. Hence $\underline{H}_{u}^{V}=\sup \underline{H}_{f}^{V}$ is finite on a dense subset of $V$. By Proposition 2.5, $\underline{H}_{u}^{V}$ is harmonic on $V$.

To prove the converse assertion, let $\left(h_{n}\right)_{n \in N}$ be an increasing sequence of harmonic functions on an open set $U$ such that $\sup h_{n}$ is finite on a dense set. Let $V$ be resolutive, sufficiently small and relatively compact in $U$. Then

$$
\sup h_{n}=\sup \underline{H}_{h_{n}}^{V}=\underline{H}_{\sup h_{n}}^{V}
$$

holds on $V$ by Lemma 2.2. By assumption, $\sup h_{n}$ is superharmonic on $U$, hence $\underline{H}_{\sup h_{n}}^{V}=\sup h_{n}$ is harmonic on $V$. By the sheaf property, $\sup h_{n}$ is harmonic on $U$.

Lemma 4.4. (1) A locally bounded hyperharmonic function is superharmonic.

(2) A hyperharmonic minorant of a superharmonic function is superharmonic.

(3) If $s_{1}, s_{2}$ are superharmonic functions, then $\inf \left(s_{1}, s_{2}\right)$ is superharmonic.

(4) A locally superharmonic function is superharmonic.

Proof. (1) Let $u \in \mathscr{U}(U)$ be locally bounded. If $V$ is resolutive, sufficiently small and relatively compact in $U$, then $u \mid \partial V$ is bounded. By Proposition 3.3, $\underline{H}_{u}^{V}$ is harmonic on $V$.

(2) Let $s$ be superharmonic on $U, u$ be hyperharmonic on $U$ and $u \leqq s$. Let further $V$ be resolutive, sufficiently small and relatively compact in $U$. Denoting again

$$
\mathscr{F}:=\{f \in \mathscr{C}(\partial V) \mid f \leqq u \text { on } \partial V\}
$$

we have $u=\sup \mathscr{F}$ on $\partial V$ and, by Lemma 3.1,

$$
\underline{H}_{u}^{V}=\underline{H}_{\text {sup } \mathscr{F}}^{V}=\sup \underline{H}_{f}^{V} \leqq \underline{H}_{s}^{V} .
$$

Since $\underline{H}_{s}^{V}$ is harmonic, $\underline{H}_{u}^{V}$ is locally bounded, hence harmonic by Proposition 2.5 .

(3) This follows immediately by Proposition 3.2 and the preceding part of this lemma.

(4) Let $u \in \mathscr{U}(U)$ be locally superharmonic and let $V$ be resolutive, sufficiently small and relatively compact in $U$. Given $x \in V$, we may take two neighbourhoods 
$W, W^{\prime}$ of $x$ such that $\mathrm{cl} W \subset W^{\prime} \subset \mathrm{cl} W^{\prime} \subset V$ such that $W$ is resolutive and $u \mid W^{\prime}$ is superharmonic on $W^{\prime}$. Hence $\underline{H}_{u}^{W}$ is harmonic. Denoting again $\mathscr{F}$ as in the proof of part (2), we get

$$
\underline{H}_{u}^{V} \mid W=\sup _{f \in \mathscr{F}}\left(\underline{H}_{f}^{V} \mid W\right)=\underline{H}_{\underline{H}_{u}^{V}}^{W} \leqq \underline{H}_{u}^{W}
$$

on $W$ by Lemma 3.1 and Proposition 3.2. Since $\underline{H}_{u}^{W}$ is harmonic on $W$, the reasoning of the preceding part (2) results in that $\underline{H}_{u}^{V}$ is harmonic on $W$. By the sheaf property, $\underline{H}_{u}^{V}$ is harmonic on $V$.

Definition 4.5. A superharmonic function $p \geqq 0$ on $X$ is a potential on $X$, if the constant function 0 is the greatest hypoharmonic minorant of $p$.

Proposition 4.6. Let $p$ be a superharmonic function on $X$. Then the following properties are equivalent:

(1) $p$ is a potential on $X$;

(2) if $u$ is hyperharmonic on $X$, then $u+p \geqq 0$ implies $u \geqq 0$.

Proof. If $u$ is hyperharmonic on $X, p$ is a potential on $X$ and $u+p \geqq 0$, then $-u \leqq p$ is a hypoharmonic minorant of $p$ hence $-u \leqq 0$ and $u \geqq 0$.

On the other hand, let $u$ be a hypoharmonic minorant of $p$ on $X$. Then $-u+p \geqq 0$ implies $-u \geqq 0$. Hence $u \leqq 0$ and $p$ is a potential on $X$.

\section{References}

[1] BAUER, H.: Harmonische Räume und ihre Potentialtheorie. - Lecture Notes in Mathematics 22. Springer-Verlag, Berlin-Heidelberg-New York, 1966.

[2] BEDFord, E., and B. A. TAYLOR: A new capacity for plurisubharmonic functions. - Acta Math. $149,1982,1-40$.

[3] Bertin, E. M. J.: Equation de Monge-Ampère et théorie du potentiel. L'axiome de faisceau. - Preprint 25, Utrecht, 1976.

[4] Bertin, E. M. J.: Fonctions convexes et théorie du potentiel. (I). - Nederl. Akad. Wetensch. Indag. Math. 41, 1979, 385-396.

[5] Bertin, E. M. J.: Fonctions convexes et théorie du potentiel. (II). - Nederl. Akad. Wetensch. Indag. Math. 41, 1979, 397-409.

[6] Brelot, M.: Lectures on potential theory. - Lectures on Mathematics 19. Tata Institute of Fundamental Research, Bombay, 1960.

[7] Constantinescu, C.: An axiomatic theory for the nonlinear Dirichlet problem. - Rev. Roumaine Math. Pures Appl. 10, 1965, 755-764.

[8] Constantinescu, C.: Le problème de Dirichlet pour des systèmes d'équations non-linéaires. Séminaire de Théorie du Potentiel, Paris, 1972-1974. Lecture Notes in Mathematics 518. Springer-Verlag, Berlin-Heidelberg-New York, 1976, 54-73.

[9] Constantinescu, C., and A. Cornea: Potential theory on harmonic spaces. - Die Grundlehren der mathematischen Wissenschaften 158, Springer-Verlag, Berlin-Heidelberg-New York, 1972. 
[10] Granlund, S., P. Lindevist, and O. Martio: Conformally invariant variational integrals. Trans. Amer. Math. Soc. 277, 1983, 43-73.

[11] Granlund, S., P. Lindqvist, and O. Martio: Note on the PWB-method in the non-linear case. - To appear.

[12] Lehtola, P.: Non-linear potential theory. - Unpublished manuscript, Jyväskylä, 1983 (Finnish).

[13] Martio, O.: Non-linear potential theory. - Summer School in Potential Theory, University of Joensuu, Department of Mathematics and Physics Report Series 5-M, Joensuu, 1983, 65-104.

University of Joensuu

Department of Mathematics

P.O. Box 111

SF-80101 Joensuu

Finland

Received 2 April 1984 